EON • Volume 14, Issue 8 - December 2021/January 2022

\title{
Message from the Editors: Our Vision for 2022
}

\section{Colin Trumbull, Lindsey Brounstein}

Published on: Dec 27, 2021

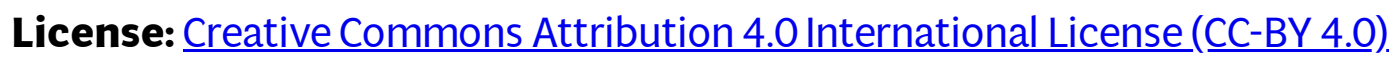




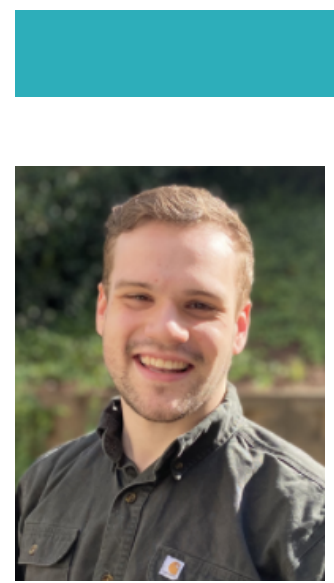

Colin

Trumbull

Staffing

Resource

Manager

J\&J Editorial,

LLC

Co-Editor-in-

Chief,

EON

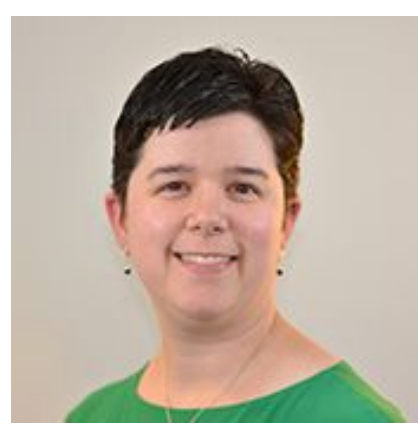

Lindsey

Brounstein

Director of

Publications,

American

Gastroenterological

Association

Co-Editor-in-Chief, EON

Ok, seriously. If whoever pressed fast forward on the universe could go ahead and bring us back to regular playback speed, that'd be great. Somehow, we find ourselves at the end of another year, and what a year it has been! Fortunately, there is at least one indirect benefit of us standing on the precipice of another year before us. That is the opportunity to reassess our position as a publication, take stock of our goals and priorities, and develop plans to achieve these goals.

We've had many discussions about our vision, and we shared a small piece of that as we unveiled our transition to PubPub as

a platform for EON. One of our greatest motivators in this move was to provide an environment that fosters and promotes direct engagement with our remarkably diverse body of readers and contributors. Ultimately, EON is primarily an outlet for the entire community at ISMTE. As such, we feel driven to build a stage for our community that gives everyone a chance to contribute their voice to the vibrant discussion we enjoy in EON and ISMTE more broadly. Given this, it is likely unsurprising that our goals for the next year center around this idea heavily. In 2022, we plan to implement some additional initiatives with the hopes of bolstering our engagement with ISMTE overall and attracting new authors and contributors to EON.

\section{Special Issues}

While EON has been fortunate to enjoy a solid core of authors, we are always eager to forge a connection with new authors. In 2020 we began a partnership with the ISMTE Early Career Committee, allowing us to connect with many new authors who have contributed to EON. We want to continue these efforts to attract new authors to EON by publishing some special issues centered around broadly appealing topics. One of the first special issues we've discussed implementing centers around the theme of leadership, decision making, and the lessons we learn when we make the wrong 
decision. We hope that a broad topic like this will attract interest from the entire range of experience evident within all of ISMTE; no matter what level of experience you have in your career, we feel that we all have something to contribute to this topic.

Look out for more information and the formal call for submissions for all of our planned special issues for 2022. We're very excited to begin implementing more special issues in the coming year and hope you will consider joining us and contributing to one of them!

\section{Multimedia Support}

Using PubPub affords us an opportunity to incorporate many more mediums than we had access to previously. We have already discussed how to utilize this functionality within PubPub to bring a more diverse range of content to our readership. Many times, we would have liked to include a video or audio file to augment an article. We're very excited to be able to support more media types in our publications to further our opportunities to engage with the community.

\section{Additional Partnerships with ISMTE Committees}

Of course, one of the staples of EON's content is our updates and highlights for ISMTE committees, giving us all a chance to stay in the know with the many committees here at ISMTE. In 2022, we would like to continue partnering with ISMTE committees, allowing us all to stay better informed of their ongoing initiatives and opportunities while also giving them a platform to speak to their priorities and perspective. Essentially, at least in part, we consider EON to be a platform for our committees, and we hope that we can continue to develop our partnership with every committee. One quick way we're planning on encouraging this partnership is to create a rotating schedule of Committee spotlights throughout the year, highlighting a select number of committees in each issue and making sure that we can hear from each of them. We think our publishing platform's additional capabilities will only strengthen this goal. For example, we believe PubPub will allow us to connect with Committees whose strategic communication might not be best suited for a written article but may benefit more from the wide range of media we will be able to incorporate into EON.

\section{Authorship Incentives, Highlighting, and Support}

We hope that it is clear from our communications over the last year that one of our ultimate goals is to foster an environment where we can highlight and celebrate all authors who donate some of their time to prepare an article for EON. Of course, we 
want to continue doing this through the open-access model we are currently experimenting with for EON. But, we also want to continue highlighting particular EON pieces that we think appeal the most broadly to our readership. We are fortunate to have been able to institute an Editorial Board to provide support and oversight to EON, and we have discussed plans to integrate them into this highlighting process. While each article will be available for anyone to read, we hope that the Editorial Board will collaborate with us to select at least one "Editor's Choice" article for each issue to shine a light on a particularly impactful piece each month.

We have also discussed the possibility of sponsorships provided by our corporate partners awarded to authors based on their submissions. While we have not yet had any formal discussions around this topic, we are excited about the possibility. We think that any strategies, tools, and methods we can implement to continue to drive the fantastic contributions we receive and reach a broader range of the ISMTE membership. Scholarships and sponsorships are an excellent way to drive this initiative, and we are looking forward to continuing these discussions in 2022.

Finally, we want to utilize social media outreach more substantially in 2022, strengthening our direct EON networks and communications to augment those that ISMTE provides more broadly. We would like to have a more significant presence within these spheres. Implementing this goal will also allow us to further highlight contributions from our authors and new initiatives we implement throughout the year.

Ultimately, we have all learned in these past two years the power that we all have to maintain our connections with one another in this virtual space. This power is immense, but it has shifted in a significant way. There are fewer opportunities for organic "eureka!" moments by a water cooler, fewer collaborative moments over lunch at an ISMTE national conference, fewer conversations in passing that turn into an exciting idea. This is not to say that these kinds of interactions no longer happen, and we are very proud of the work ISMTE, in particular, has done to foster these kinds of interaction in our virtual space. Still, we think this has also highlighted the need to do these things deliberately. We need to actively plan for new opportunities to interact and collaborate with one another, and we need to build our network and community with that deliberate action in mind. We hope that our pointed goals for 2022 will allow us to contribute to ISMTE's community deliberately, and we are so excited for the opportunity for you all to join us on this journey in the year to come. 